\title{
Solution of Abel Integral Equation Using Differential Transform Method
}

Subhabrata Mondal ${ }^{1}$ and B.N. Mandal ${ }^{2}$

${ }^{1}$ Department of Applied Mathematics, University of Calcutta, 92, A.P.C. Road, Kolkata-700009, India subhamath644@gmail.com

${ }^{2}$ Physics and Applied Mathematics Unit, Indian Statistical Institute, 203, B.T Road, Kolkata-700108, India bnm2006@rediffmail.com

\begin{abstract}
The application of fractional differential transform method, developed for differential equations of fractional order, are extended to derive exact analytical solutions of fractional order Abel integral equations. The fractional integrations are described in the Riemann-Liouville sense and fractional derivatives are described in the Caputo sense. Abel integral equation occurs in the mathematical modeling of various problems in physics, astrophysics, solid mechanics and applied sciences. An analytic technique for solving Abel integral equation of first kind by the proposed method is introduced here. Also illustrative examples with exact solutions are considered to show the validity and applicability of the proposed method. Abel integral equation, Differential transform method, Fractional differential transform method.
\end{abstract}

Indexing terms/Keywords: Abel Integral Equation, Differential Transform Method, Fractional Differential Transform Method.

Language: English

Date of Submission: 2018-03-06

Date of Acceptance: 2018-03-20

Date of Publication: 2018-03-30

ISSN: 2347-1921

Volume: 14 Issue: 01

Journal: Journal of Advances in Mathematics

Website: https://cirworld.com

This work is licensed under a Creative Commons Attribution 4.0 International License.

\section{Introduction}

It is well-known fact that many problems in various branches of engineering and applied sciences are converted to integral equations. Abel integral equation is directly derived from a problem of mechanics. Abel integral equations of first kind are of two types given by

$$
\int_{a}^{x} \frac{\phi(t)}{(x-t)^{\frac{1}{2}}} d t=f(x), x>a(f(a)=0)
$$

and 


$$
\int_{x}^{b} \frac{\phi(t)}{(t-x)^{\frac{1}{2}}} d t=f(x), a<x<b(f(b)=0) .
$$

A generalisation of (1.1) and (1.2) is

$$
\int_{a}^{x} \frac{\phi(t)}{(x-t)^{\alpha}} d t=f(x), x>a(f(a)=0)
$$

and

$$
\int_{x}^{b} \frac{\phi(t)}{(t-x)^{\alpha}} d t=f(x), a<x<b(f(b)=0)
$$

where $\alpha$ is a real constant such that $0<\alpha<1, f(x)$ is a known function and $\phi(x)$ is an unknown function to be determined. Exact solutions of these equations are well-known and are given by

$$
\phi(x)=\frac{\sin \pi \alpha}{\pi} \frac{d}{d x}\left[\int_{a}^{x} \frac{f(t)}{(x-t)^{1-\alpha}} d t\right]
$$

and

$$
\phi(x)=-\frac{\sin \pi \alpha}{\pi} \frac{d}{d x}\left[\int_{x}^{b} \frac{f(t)}{(t-x)^{1-\alpha}} d t\right]
$$

respectively.

Mandal et al [1] solved a system of generalized Abel integral equations by using fractional calculus. Yousefi [2] obtained numerical solution of Abel integral equation by using Legendre wavelets. Liu and Tao [3] used mechanical quadrature methods for solving first kind Abel integral equations. Chakrabarti [4] obtained the solution of generalized Abel integral equation by a direct function theoretic method. De et al [5] reinvestigated the water wave scattering problem involving two submerged plane thin vertical barriers by an approach leading to the problem of solving a system of Abel integral equations. Pandey and Mandal [6] obtained numerical solution of a system of generalized Abel integral equations using Bernstein polynomials. Dixit et al [7] solved generalized Abel integral equation by using Bernstein operational matrix. Pandey et al [8] used collocation method for the solution of generalized Abel integral equations.

Abel integral equation can be considered as a fractional integral equation, where the order of the fractional integral equations (1.3) and (1.4) are $(1-\alpha)$. Here fractional and generalized differential transform methods have been employed to solve the integral equations (1.3) and (1.4) wherein the result

$$
\int_{0}^{x} \frac{t^{\beta k}}{(x-t)^{\alpha}} d t=\frac{\Gamma(1+\beta k) \Gamma(1-\alpha)}{\Gamma(2+\beta k-\alpha)} x^{1+\beta k-\alpha}, \quad 0<\alpha<1 .
$$

has been used. 


\section{Analysis Of The Fractional Differential Transform Method}

Arikoglu and Ozkol [9] introduced the fractional differential transform method (FDTM) for solving fractional differential equations. For convenience, in this section a short review of the FDTM is presented with some preliminary concepts and definitions.

The fractional differential transform of the $k$ th derivative of the analytic function $f(x)$ evaluated at the point $x_{0}$ is defined as

$$
F(k)= \begin{cases}0 & \frac{k}{q} \notin \mathbf{Z}^{+}, \\ \left.\frac{1}{\left(\frac{k}{q}\right) !\left(\frac{d^{\frac{k}{q}}}{d x^{\frac{k}{q}}} f(x)\right.}\right)_{x=x_{0}} & , \frac{k}{q} \in \mathbf{Z}^{+}\end{cases}
$$

where $q$ is the order of fraction of the fractional equation.

There are several approaches to the generalization of the notion of differentiation to fractional orders. The fractional differentiation in Riemann-Liouville sense is defined by

$$
D_{x_{0}}^{\beta} f(x)=\frac{1}{\Gamma(m-\beta)} \frac{d^{m}}{d x^{m}}\left[\int_{x_{0}}^{x} \frac{f(t)}{(x-t)^{1+\beta-m}} d t\right]
$$

for $m-1 \leq \beta<m, m \in \mathbf{Z}^{+}, x>x_{0}$ and $\beta$ is the order of the corresponding fractional equation.

Concerning the practical applications encountered in various branches of science, the fractional initial conditions are frequently not available, and it may not be clear what their physical meaning is. Therefore, the definition in Eq. (2.2) should be modified to deal with integer ordered initial conditions in Caputo sense [10] as follows :

$$
\begin{aligned}
& D_{*_{x_{0}}}^{\beta} f(x)=D_{x_{0}}^{\beta}\left[f(x)-\sum_{k=0}^{m-1} \frac{1}{k !}\left(x-x_{0}\right)^{k} f^{(k)}\left(x_{0}\right)\right] \\
& =\frac{1}{\Gamma(m-\beta)} \frac{d^{m}}{d x^{m}}\left[\int_{x_{0}}^{x} \frac{f(t)-\sum_{k=0}^{m-1} \frac{1}{k !}\left(t-x_{0}\right)^{k} f^{(k)}\left(x_{0}\right)}{(x-t)^{1+\beta-m}} d t\right]
\end{aligned}
$$

for $k=0,1,2, \ldots .(q \beta-1)$.

Thus the fractional differential inverse transform of $F(k)$ evaluated at the point $x_{0}$ is defined as 


$$
f(x)=\sum_{k=0}^{\infty} F(k)\left(x-x_{0}\right)^{\frac{k}{q}} \text {, }
$$

which implies that the concept of fractional differential transform is derived from fractional power series expansion. In practical application, the function $f(x)$ can be approximated by the finite series

$$
f(x) \equiv \sum_{k=0}^{n} F(k)\left(x-x_{0}\right)^{\frac{k}{q}} .
$$

From definitions (2.1) and (2.3), Some basic properties of the fractional differential transform are as follows

P1 : If $f(x)=g(x) \pm h(x)$, then $F(k)=G(k) \pm H(k)$.

$\mathbf{P 2}:$ If $f(x)=g(x) h(x)$, then $F(k)=\sum_{l=0}^{k} G(l) H(k-l)$.

P3 : If $f(x)=c g(x)$, then $F(k)=c G(k)$.

$\mathbf{P 4}$ : If $f(x)=x^{p}$, then $F(k)=\delta(k-q p)= \begin{cases}1 & \text { if } k=q p \\ 0 & \text { if } k \neq q p .\end{cases}$

P5 : If $f(x)=D_{*_{x_{0}}}^{\beta} g(x)$, then $F(k)=\frac{\Gamma\left(\beta+1+\frac{k}{q}\right)}{\Gamma\left(1+\frac{k}{q}\right)} G(k+\beta q)$.

P6 : If $f(x)=\int_{x_{0}}^{x} g(t) d t$, then $F(k)=\frac{q G(k-q)}{k}, k \geq q$.

P7 : If $f(x)=g(x) \int_{x_{0}}^{x} h(t) d t$, then $F(k)=q \sum_{k_{1}=q}^{k} \frac{H\left(k_{1}-q\right)}{k_{1}} G\left(k-k_{1}\right), k \geq q$.

\section{Solution Of Abel Integral Equation Of First Kind}

In this section Abel integral equations (1.3) and (1.4) are solved by using fractional differential transform method.

By using the transformations

$$
t-a=z, x-a=y, \phi(z+a)=\phi_{1}(z), f(y+a)=f_{1}(y),
$$

equation (1.3) becomes 


$$
\int_{0}^{y} \frac{\phi_{1}(z)}{(y-z)^{\alpha}} d z=f_{1}(y), y>0, f_{1}(0)=0 .
$$

Here $(1-\alpha)$ is the fractional order of Integral equation (3.1).

Let $\alpha=\frac{p}{q}$, where $p$ and $q$ are both positive integers and $p<q, p, q$ have no common factor.

Here $q$ is the order of fraction of the fractional integral equation (3.1).

Let

$$
\phi_{1}(x)=\sum_{k=0}^{\infty} \Phi_{1}(k) x^{\frac{k}{q}}
$$

and

$$
f_{1}(x)=\sum_{k=0}^{\infty} F_{1}(k) x^{\frac{k}{q}}
$$

where $\Phi_{1}(k)$ and $F_{1}(k)$ are the fractional differential transforms of $\phi_{1}(x)$ and $f_{1}(x)$ respectively.

Use of equation (3.2) and the result (1.7) in equation (3.1) provides

$$
\sum_{k=0}^{\infty} \Phi_{1}(k)\left[\frac{\Gamma\left(1+\frac{k}{q}\right) \Gamma\left(1-\frac{p}{q}\right)}{\Gamma\left(2+\frac{k}{q}-\frac{p}{q}\right)}\right] y^{1+\frac{k}{q}-\frac{p}{q}}=f_{1}(y) .
$$

Taking fractional differential transform on both sides, (3.4) produces

$$
\sum_{k=0}^{\infty} \Phi_{1}(k)\left[\frac{\Gamma\left(1+\frac{k}{q}\right) \Gamma\left(1-\frac{p}{q}\right)}{\Gamma\left(2+\frac{k}{q}-\frac{p}{q}\right)}\right] \delta[m-(q+k-p)]=F_{1}(m) .
$$

This gives

$$
\Phi_{1}(m)=F_{1}[m-(p-q)] \frac{\Gamma\left[\frac{m-p}{q}+2\right]}{\Gamma\left[\frac{m+q}{q}\right] \Gamma\left[1-\frac{p}{q}\right]},
$$

which can be written as 


$$
\Phi_{1}(m)=\frac{1}{\Gamma\left(1-\frac{p}{q}\right)} \sum_{k=0}^{\infty} F_{1}(k) \frac{\Gamma\left[1+\frac{k}{q}\right]}{\Gamma\left[1+\frac{k}{q}+\frac{p}{q}\right]}\left(\frac{k}{q}+\frac{p}{q}\right) \delta[m-(k+p-q)] .
$$

Taking inverse fractional differential transform on both sides of equation (3.6), one obtains

$$
\begin{aligned}
& \phi_{1}(y)=\frac{1}{\Gamma\left(1-\frac{p}{q}\right)} \sum_{k=0}^{\infty} F_{1}(k) \frac{\Gamma\left[1+\frac{k}{q}\right]}{\Gamma\left[1+\frac{k}{q}+\frac{p}{q}\right]}\left(\frac{k}{q}+\frac{p}{q}\right) y^{\frac{k}{q}+\frac{p}{q}-1} \\
& =\frac{1}{\Gamma\left(1-\frac{p}{q}\right) \Gamma\left(\frac{p}{q}\right)} \frac{d}{d y}\left[\sum_{k=0}^{\infty} F_{1}(k) \frac{\Gamma\left[1+\frac{k}{q}\right] \Gamma\left[1-\frac{q-p}{q}\right]}{\Gamma\left[2+\frac{k}{q}-\frac{q-p}{q}\right]} y^{1+\frac{k}{q}-\left(\frac{q-p}{q}\right)}\right] \\
& =\frac{1}{\Gamma\left(1-\frac{p}{q}\right) \Gamma\left(\frac{p}{q}\right)} \frac{d}{d y}\left[\sum_{k=0}^{\infty} F_{1}(k) \int_{0}^{y} \frac{z^{\frac{k}{q}}}{(y-z)^{\frac{q-p}{q}}} d z\right] \\
& =\frac{\sin \frac{p}{q} \pi}{\pi} \frac{d}{d y}\left[\int_{0}^{y} \frac{f_{1}(z)}{(y-z)^{1-\frac{p}{q}}} d z\right] \\
& =\frac{\sin \alpha \pi}{\pi} \frac{d}{d y}\left[\int_{0}^{y} \frac{f_{1}(z)}{(y-z)^{1-\alpha}} d z\right] \text {. }
\end{aligned}
$$

Noting that $\phi_{1}(y)=\phi(y+a), f_{1}(z)=f(z+a)$ and back substitution of $z=t-a, y=x-a$ in produces the solution of the Abel integral equation (1.3) as given by (1.5).

Similarly, if one uses the transformations

$$
b-t=z, b-x=y, \phi(b-z)=\phi_{2}(z), f(b-y)=f_{2}(y)
$$

then (1.4) becomes

$$
\int_{0}^{y} \frac{\phi_{2}(z)}{(y-z)^{\alpha}} d z=f_{2}(y), y>0, f_{2}(0)=0 .
$$

In a similar way, one can obtain

$$
\phi_{2}(y)=\frac{\sin \alpha \pi}{\pi} \frac{d}{d y}\left[\int_{0}^{y} \frac{f_{2}(z)}{(y-z)^{1-\alpha}} d z\right]
$$


and by using $\phi_{2}(y)=\phi(b-y), f_{2}(z)=f(b-z)$ and back substitution of $z=b-t, y=b-x$ into (3.8) produces the solution of the Abel integral equation (1.4) as given by (1.6).

\section{Illustrative Examples}

Example 1. Consider the Abel integral equation of second kind

$$
\phi(x)+\int_{0}^{x} \frac{\phi(t)}{(x-t)^{\frac{1}{2}}} d t=2 \sqrt{x},
$$

which has $\phi(x)=1-e^{\pi x} \operatorname{erfc}(\sqrt{\pi x})$ as the exact solution.

Here the order of fraction of equation (4.1) is 2.

Using basic properties of the fractional differential transform, the fractional differential transform of $f(x)$ is obtained as

$$
F(m)=2 \delta(m-1)
$$

so that

$$
F(m)= \begin{cases}2 & \text { for } m=1 \\ 0 & , \text { otherwise }\end{cases}
$$

By using (3.5), the fractional differential transform of equation (4.1) is

$$
\begin{aligned}
& \Phi(m)+\sum_{k=0}^{\infty} \Phi(k) \frac{\Gamma\left(\frac{1}{2}\right) \Gamma\left(1+\frac{k}{2}\right)}{\Gamma\left(\frac{3}{2}+\frac{k}{2}\right)} \delta[m-(1+k)]=F(m) \\
& \text { so that } \Phi(m)+\Phi(m-1) \frac{\Gamma\left(\frac{1}{2}\right) \Gamma\left(1+\frac{m-1}{2}\right)}{\Gamma\left(\frac{3}{2}+\frac{m-1}{2}\right)}=F(m) .
\end{aligned}
$$

Putting $m=0,1,2,3, \ldots$. the following system of linear equations are obtained

$$
\begin{gathered}
\Phi(0)=0 \\
\Phi(1)+2 \Phi(0)=2
\end{gathered}
$$




$$
\begin{aligned}
& \Phi(2)+\frac{\pi}{2} \Phi(1)=0 \\
& \Phi(3)+\frac{4}{3} \Phi(2)=0 \\
& \Phi(4)+\frac{3 \pi}{8} \Phi(3)=0
\end{aligned}
$$

... and so on.

This has the following solution

$$
\begin{gathered}
\Phi(0)=0=0 \\
\Phi(1)=2=\frac{(-1)^{1-1}(\pi)^{\frac{1}{2}}}{\Gamma\left(1+\frac{1}{2}\right)} \\
\Phi(2)=-\pi=\frac{(-1)^{2-1}(\pi)^{\frac{2}{2}}}{\Gamma\left(1+\frac{2}{2}\right)} \\
\Phi(3)=\frac{4 \pi}{3}=\frac{(-1)^{3-1}(\pi)^{\frac{3}{2}}}{\Gamma\left(1+\frac{3}{2}\right)}
\end{gathered}
$$

Thus

$$
\Phi(k)= \begin{cases}0 & k=0 \\ \frac{(-1)^{k-1}(\pi)^{\frac{k}{2}}}{\Gamma\left(1+\frac{k}{2}\right)} & k=1,2,3, \ldots\end{cases}
$$

Now, use of inverse fractional differential transform one gets 


$$
\begin{aligned}
& \phi(x)=\sum_{k=0}^{\infty} \Phi(k) x^{\frac{k}{2}} \\
& =\Phi(1) x^{\frac{1}{2}}+\Phi(2) x+\Phi(3) x^{\frac{3}{2}}+\Phi(4) x^{2}+\ldots \\
& =2 \sqrt{x}-\pi x+\frac{4 \pi}{3} x^{\frac{3}{2}}-\frac{\pi^{2} x^{2}}{2}+\ldots . . \\
& =\sum_{n=1}^{\infty} \frac{(-1)^{n-1}(\pi x)^{\frac{n}{2}}}{\Gamma\left(1+\frac{n}{2}\right)} \\
& =1-E_{\frac{1}{2}}(-\sqrt{\pi x}) \\
& =1-e^{\pi x} \operatorname{erfc}(\sqrt{\pi x}),
\end{aligned}
$$

which is the exact solution of equation (4.1).

Example 2. Consider the Abel integral equation of second kind

$$
\phi(x)+\int_{0}^{x} \frac{\phi(t)}{(x-t)^{\frac{1}{4}}} d t=\frac{1}{2 \sqrt{2}} \pi x+x^{\frac{1}{4}},
$$

which has $\phi(x)=x^{\frac{1}{4}}$ as the exact solution.

Here the order of fraction of equation (4.2) is 4 .

Using basic properties of fractional differential transform, one obtains the fractional differential transform of $f(x)$ as

$$
F(m)=\frac{1}{2 \sqrt{2}} \pi \delta(m-4)+\delta(m-1)
$$

Therefore,

$$
F(m)=\left\{\begin{array}{lcc}
\frac{1}{2 \sqrt{2}} \pi & \text { for } & m=4 \\
1 & , & m=1 \\
0 & & \text { otherwise }
\end{array}\right.
$$

By using (3.5), one gets the fractional differential transform of equation (4.2) as 


$$
\Phi(m)+\sum_{k=0}^{\infty} \Phi(k) \frac{\Gamma\left(\frac{3}{4}\right) \Gamma\left(1+\frac{k}{4}\right)}{\Gamma\left(\frac{7}{4}+\frac{k}{4}\right)} \delta[m-(k+3)]=F(m)
$$

so that

$$
\Phi(m)+\Phi(m-3) \frac{\Gamma\left(\frac{3}{4}\right) \Gamma\left(1+\frac{m-3}{4}\right)}{\Gamma\left(\frac{7}{4}+\frac{m-3}{4}\right)}=F(m) .
$$

Putting $m=0,1,2,3, \ldots . \quad$, one obtains

$$
\begin{gathered}
\Phi(0)=0 \\
\Phi(1)=1 \\
\Phi(2)=0 \\
\Phi(3)+\Phi(0)=0 \\
\Phi(4)+\frac{\pi}{2 \sqrt{2}} \Phi(1)=\frac{\pi}{2 \sqrt{2}} \\
\Phi(k)=\left\{\begin{array}{l}
1 \quad \text { for } \quad k=1 \\
0 \quad, \quad \text { otherwise }
\end{array}\right.
\end{gathered}
$$

so that

Now, using inverse fractional differential transform one finds

$$
\begin{aligned}
& \phi(x)=\sum_{k=0}^{\infty} \Phi(k) x^{\frac{k}{4}} \\
& =\Phi(1) x^{\frac{1}{4}} \\
& =x^{\frac{1}{4}}
\end{aligned}
$$


which is the exact solution of equation (4.2).

Example 3. Consider the Abel integral equation of the first kind

$$
\int_{0}^{x} \frac{\phi(t)}{(x-t)^{\frac{1}{3}}} d t=\frac{2}{3 \sqrt{3}} \pi x
$$

which has $\phi(x)=x^{\frac{1}{3}}$ as the exact solution.

Here the order of fraction of Eq. (4.3) is 3 .

Using basic properties of fractional differential transform, one obtains the fractional differential transform of $f(x)=\frac{2}{3 \sqrt{3}} \pi x$ as

$$
F(m)=\frac{2}{3 \sqrt{3}} \pi \delta(m-3) .
$$

Therefore,

$$
F(m)= \begin{cases}\frac{2}{3 \sqrt{3}} \pi & m=3 \\ 0 & , \text { otherwise }\end{cases}
$$

By using (3.5), one gets the fractional differential transform of equation (4.3) as

$$
\begin{gathered}
\sum_{k=0}^{\infty} \Phi(k) \frac{\Gamma\left(\frac{2}{3}\right) \Gamma\left(1+\frac{k}{3}\right)}{\Gamma\left(\frac{5}{3}+\frac{k}{3}\right)} \delta[m-(2+k)]=\frac{2}{3 \sqrt{3}} \pi \delta(m-3) \\
\text { so that } \Phi(m-2) \frac{\Gamma\left(\frac{2}{3}\right) \Gamma\left(1+\frac{m-2}{3}\right)}{\Gamma\left(\frac{5}{3}+\frac{m-2}{3}\right)}=\frac{2}{3 \sqrt{3}} \pi \delta(m-3) .
\end{gathered}
$$

Putting $m=2,3, \ldots$ we obtain 


$$
\Phi(k)= \begin{cases}1 & k=1 \\ 0 & , \text { otherwise }\end{cases}
$$

Now, we have from inverse fractional differential transform

$$
\begin{aligned}
& \phi(x)=\sum_{k=0}^{\infty} \Phi(k) x^{\frac{k}{3}} \\
& =\Phi(1) x^{\frac{1}{3}} \\
& =x^{\frac{1}{3}}
\end{aligned}
$$

which is the exact solution of equation (4.3).

\section{References}

[1] N. Mandal, A. Chakrabarti, B. N. Mandal, Solution of a system of generalized Abel integral equations using fractional calculus, Appl. Math. Lett. 9 No. 3(1996) 1-4.

[2] S.A. Yousefi, Numerical solution of Abel's integral equation by using Legendre wavelets, Appl. Math. Comp. 175(2006) 574-580.

[3] Y. Liu, L. Tao, Mechanical quadrature methods and their extrapolation for solving first kind Abel integral equations, Jour. Comput. Appl. Math. 201(2007) 300-313.

[4] A. Chakrabarti, Solution of the Generalized Abel Integral Equations, J. Integral Equations Applications 20(2008) 1-11.

[5] S. De, B. N. Mandal, A. Chakrabarti, Water wave scattering by two submerged plane vertical barriers - Abel integral equations approach, J. Engng. Math. 65(2009) 75-87.

[6] R. K. Pandey, B.N. Mandal, Numerical solution of a system of Generalized Abel Integral Equation using Bernstein polynomials, J. Adv. Res. Sci. Comput. 2(2010) 44-53.

[7] S. Dixit, R. K. Pandey, S. Kumar, O.P. Singh, Solution of the Generalized Abel Integral Equation by using Almost Bernstein Operational Matrix, American Jour. Comput. Math. 1(2011) 226-234.

[8] R. K. Pandey, S. Sharma, K. Kumar, Collocation method for Generalized Abel's Integral Equations, Jour. Comput. Appl. Math. 302(2016) 118-128.

[9] A. Arikoglu, I. Ozkol, Solution of fractional differential equations by using differential transform method, Chaos, Solitons and Fractals 34(2007) 1473-1481.

[10] M. Caputo, Linear models of dissipation whose Q is almost frequency independent, Part II. J Roy Austral Soc 13(1967) 529-539. 